\title{
COMPARAÇÃO DA FORÇA MUSCULAR MÁXIMA ENTRE IDOSAS PRATICANTES DE TREINAMENTO DE FORÇA E USUÁRIAS DE ACADEMIAS DA TERCEIRA IDADE
}

\author{
Valdemar da $\operatorname{COSTA}^{1}$ \\ Fernando POSSAMAI ${ }^{2}$
}

José Roberto Andrade do NASCIMENTO JÚNIOR ${ }^{3}$

Mateus Dias ANTUNES ${ }^{4}$

Daniel Vicentini de OLIVEIRA ${ }^{5}$

\begin{abstract}
${ }^{1}$ Profissional de Educação Física pela Faculdade Metropolitana de Maringá - FAMMA, Maringá, PR, Brasil. valdemarvdr@gmail.com.

${ }^{2}$ Profissional de Educação Física. Universidade Estadual de Maringá - UEM, Maringá, PR, Brasil. fernando@personal.pro.br.

${ }^{3}$ Profissional de Educação Física. Mestre e Doutor em Educação Fisica pela Universidade Estadual de Maringá UEM, Maringá, PR, Brasil. jroberto.jrs01@gmail.com.

${ }^{4}$ Fisioterapeuta, discente do Programa de Pós Graduação em Promoção da Saúde do Centro Universitário de Maringá - UNICESUMAR (Bolsista CAPES), Maringá, PR, Brasil. mateus_antunes03@ hotmail.com

${ }^{5}$ Fisioterapeuta e Profissional de Educação Física. Mestre em Promoção da Saúde. Doutorando do Programa de Pós Graduação em Gerontologia da Universidade Estadual de Campinas - UNICAMP, Campinas, SP, Brasil. d.vicentini@hotmail.com
\end{abstract}

Recebido em: 14/11/2016 - Aprovado em: 05/06/2017 - Disponibilizado em: 01/07/2017

\section{RESUMO}

Objetivo: comparar a força muscular máxima de idosas praticantes de exercícios resistidos e de usuárias das Academias da Terceira Idade (ATIs). Método: Para tal, foi utilizada uma pesquisa do tipo quantitativa, observacional e transversal, na qual a amostra foi composta por 60 idosas, divididas em dois grupos, sendo o Grupo TF (treinamento de força) composto de 30 idosas praticantes exclusivamente de exercícios resistidos e, o Grupo ATI, de 30 idosas usuárias das ATIs, sem apresentarem, nos últimos seis meses, histórico de lesões articulares ou musculares. A força muscular máxima foi avaliada por meio do teste de 1RM, o qual foi realizado em uma única sessão, nos exercícios: rosca direta no crossover, tríceps pulley, cadeira extensora e mesa flexora. Resultados: a média de idade das idosas foi de 64,13 anos. Os resultados apresentaram diferença significativa $(\mathrm{p}<0,01)$ entre os grupos nos exercícios tríceps pulley, cadeira extensora, rosca direta no crossover, no qual as idosas do Grupo 1 apresentaram maior força muscular máxima em todos exercícios avaliados. Conclusão: é significativamente maior a força muscular máxima de idosas praticantes de exercícios resistidos do que de idosas usuárias das ATIs, não praticantes de exercício físico. Sugere-se o estímulo e aderência de idosas em um programa treinamento orientado, estruturado, organizado e planejado para a manutenção da independência e habilidades físicas durante o processo de envelhecimento.

Palavras-chave: Envelhecimento; Exercício físico; Capacidade física; Treinamento de força.

\begin{abstract}
Objective: To compare the maximum muscular strength of elderly women practicing resistance exercises and users of the Third age gym (TAG). Method: A quantitative, observational and cross-sectional study was used in which the sample consisted of 60 elderly women, divided into two groups, the ST group (strength training) composed of 30 elderly women practicing resistance exercises, and the TAG Group of 30 elderly women who as without presenting, in the last six months, a history of joint or muscle injuries. The maximal muscle strength was evaluated through the 1RM test, which was performed in a single session, in the exercises: direct crossover, triceps pulley, extensor chair and flexor table. Results: the mean age of the elderly was 64.13 years. The results presented a significant difference $(\mathrm{p}<0.01)$ between the groups in the triceps pulley, extensor chair, and direct crossover exercises, in which the elderly women in Group 1 had the highest maximum muscle strength in all the exercises evaluated. Conclusion: the maximal muscular strength of elderly women who practice resisted
\end{abstract}


exercises is significantly greater than that of elderly users of ITAs, who do not exercise. It is suggested the stimulation and adherence of the elderly in a program oriented, structured, organized and planned training for the maintenance of independence and physical abilities during the aging process.

Keywords: Aging; Physical exercise; Physical capacity; Strength training.

\section{INTRODUÇÃO}

O envelhecimento no Brasil está acontecendo em ritmo acelerado, envolvendo vários aspectos, como aumento do número de doenças crônicas, diminuição do estilo de vida ativo, dentre outros. Estima-se que a população de idosos no país será superior ao de crianças até o ano de 2025. O processo de envelhecimento varia de indivíduo para indivíduo (CAETANO, 2006), sedo caracterizado como dinâmico progressivo e irreversível, ligado intimamente a alterações biológicas (físicas e fisiológicas), psíquicas e sociais (BRITO; LITVOC, 2004). Uma das mais principais alterações que ocorre durante esse processo é a diminuição da área muscular, cerca de $40 \%$, que leva à redução da força muscular e consequentemente ao declínio da mobilidade e da capacidade funcional do idoso (MATSUDO et al., 2000; WIECHMANN; RUZENE; NAVEGA, 2013).

Dentre as alterações físicas, a perda de massa muscular e força é um dos mais sérios problemas do envelhecimento. De acordo com Prado, Teixeira e Langa (2010) o número total de fibras musculares diminui, e ocorre atrofia, principalmente das fibras musculares do tipo II, as fibras de contração rápida, que são responsáveis pelo desenvolvimento da força. Há entre a $6^{\mathrm{a}}$ e a $7^{\mathrm{a}}$ década de vida um declínio de $15 \%$ e nas décadas subsequentes, $30 \%$. Esses achados podem ser potencializados pela inatividade física: há uma redução de $2 \%$ a $3 \%$ de massa muscular em indivíduos sedentários por ano. Indivíduos que apresentam redução de massa muscular e, consequentemente, de força, terão prejuízos na execução das atividades diárias (MATSUDO et al., 2000; WIECHMANN; RUZENE; NAVEGA, 2013).

A redução de massa muscular tem como principal característica a diminuição do desempenho motor, podendo ocorrer antes do surgimento das manifestações clínicas e do prejuízo funcional, conferindo aos idosos, maior probabilidade de quedas, fraturas, incapacidade, dependência, hospitalizações recorrentes e aumento da mortalidade (MARTIN; NEBULONI; NAJAS, 2012). No entanto, a manutenção de um estilo de vida ativo por meio da realização regular de exercício físico aeróbico e de força contribui para um envelhecimento mais saudável, que se 
caracteriza, entre outros aspectos, por níveis de aptidão que se relacionam com menor risco de morbidade, mortalidade, previne quedas e dependência funcional (PADOIN et al., 2010).

Os exercícios para desenvolvimento de força e resistência são estratégias de promoção de saúde mais recomendadas pelo American College of Sports Medicine. Nos idosos, os treinamentos resistidos de força colaboram para melhorar o tônus, a força e a massa muscular e ajuda a preservar e a aprimorar a qualidade física, e assim, diminuir riscos prováveis déficits (PADOIN et al., 2010; AGUIAR et al., 2014).

Os exercícios resistidos atuam na prevenção e tratamento da osteoporose, melhoria das doenças degenerativas osteoarticulares, possibilitando melhor desempenho nas atividades aeróbicas, por conseguinte, incremento no VO2 máx. Com isso, haverá aumento do equilíbrio e coordenação, independência, autoestima, diminuição das quedas, provocando menor prevalência de depressão, ou melhor, o seu controle, invertendo o sentido do ciclo, no caminho da diminuição da inatividade física (FREITAS; KOPILLER, 2006).

Embora existam dados brasileiros sobre força muscular máxima em idosas praticantes de exercícios resistidos, e de outras variáveis em idosas usuárias das Academias da Terceira Idade, não há estudos comparando a força muscular máxima entre estas duas populações. Posto isso, o presente estudo teve como objetivo comparar a força muscular máxima de idosas praticantes de exercícios resistidos e idosas usuárias das ATIs.

\section{METODOLOGIA}

O presente estudo tem caráter quantitativo, observacional e transversal, e foi aprovado pelo Comitê de Ética em Pesquisa (CEP) do Centro Universitário de Maringá (UNICESUMAR) por meio do protocolo 1440930/2015. A amostra foi selecionada de forma não probabilística, por conveniência, sendo composta por 60 idosas com idade entre 60 a 70 anos, dividida em dois grupos: 30 idosas praticantes de treinamento de força (Grupo TF) e 30 idosas usuárias das ATIs (Grupo ATI).

O grupo TF era composta de idosas $(64,13 \pm 5,27$ anos $)$ praticantes exclusivamente de treinamento de força em uma academia de musculação do município de Maringá, há pelo menos seis meses, e duas vezes semanais de prática. As idosas apresentaram massa corporal média de $67,12 \pm 8,72 \mathrm{~kg}$ e estatura média de 1,62 \pm 0,05 metros. No Grupo ATI, foram inclusas idosas $(67,97 \pm 7,03$ anos $)$ usuárias de uma das cinco ATIs mais movimentadas do município, segundo a Secretaria de Esportes e Lazer, não 
praticantes de exercícios físicos. As idosas apresentaram massa corporal média de $69,69 \pm 10,51 \mathrm{~kg}$ e estatura média de 1,61 $\pm 0,06$ metros. Idosas com dificuldades motoras e neurológicas perceptíveis para realização dos testes e para responder aos questionamentos dos pesquisadores, foram excluídas, assim como as que informaram ter sofrido lesão muscular e/ou articular nos últimos três meses.

Foi utilizado um questionário sociodemográfico, elaborado pelos próprios autores, com questões referentes à idade, tempo de prática da modalidade de exercício resistido ou uso das ATIs. O peso foi avaliado por meio de balança digital da marca G-Life e a altura por meio de estadiômetro da marca MD-2M. O instrumento utilizado para a avaliação da força muscular máxima foi o Teste de repetições por carga máxima (TRCM), por apresentarem menores riscos de lesão ou desconforto muscular, muito usado em crianças, idosos e iniciantes em treinamento resistido. Este teste determina a quantidade de repetições que o indivíduo consegue executar com um peso prédeterminado, possibilitando o mesmo repetir mais vezes o movimento, até que chegue à fadiga total (GUEDES; GUEDES, 2006).

As participantes foram orientadas sobre o estudo, todas receberam e assinaram um termo de consentimento livre e esclarecido (TCLE) de participação na pesquisa de cunho científico. Todas as idosas foram convidadas a irem até uma academia para que fosse realizado o TRCM. Cada idosa executou nos equipamentos, cadeira extensora, mesa flexora, rosca direta no crossover e tríceps pulley, a maior quantidade possível de repetições. Os resultados do referido teste foram registrados pelos pesquisadores e passados para um programa matemático no Software Microsoft Excel 2013, dentro deste, utilizou-se de cálculo e protocolo de Guedes e Guedes (2006) o qual se converteu os dados pra o teste de 1RM. Para a realização desses procedimentos, esteve presente o pesquisador, o supervisor da academia e, convidado a participar dessa coleta, por motivos de segurança, um médico para que se pudesse garantir a integridade pessoal do voluntário. A coleta de dados foi realizada entre julho e setembro de 2015.

Para análise dos dados, inicialmente foi aplicado o teste Kolmogorov-Smirnov para a verificação da normalidade das variáveis em estudo. Como os dados apresentaram distribuição normal, foi utilizada a Média (x) e o Desvio-Padrão (dp) para a caracterização dos resultados. Para comparar as variáveis entre os dois grupos, utilizou-se o teste $\mathrm{t}$ de student independente. A significância adotada foi 
de $p<0,05$. Os dados foram analisados no software SPSS versão 23.0.

\section{RESULTADOS}

A Tabela 1 apresenta a comparação da força máxima de idosas praticantes de TF e ATI da cidade de Maringá-PR.

Tabela 1 - Comparação da força máxima entre as idosas praticantes de TF e ATI da cidade de Maringá-PR

\begin{tabular}{|c|c|c|c|}
\hline \multirow{2}{*}{ Variáveis } & Grupo TF & $\begin{array}{c}\text { Grupo } \\
\text { ATI }\end{array}$ & \multirow{2}{*}{$\boldsymbol{P}$} \\
\hline & $x \pm d p$ & $x \pm d p$ & \\
\hline Tríceps Pulley & $\begin{array}{c}40.73 \pm \\
9.78\end{array}$ & $\begin{array}{c}30.07 \pm \\
7.25\end{array}$ & $0.001 *$ \\
\hline $\begin{array}{l}\text { Cadeira } \\
\text { Extensora }\end{array}$ & $\begin{array}{c}38.57 \pm \\
11.36\end{array}$ & $\begin{array}{c}26.97 \pm \\
7.46\end{array}$ & $0.001 *$ \\
\hline $\begin{array}{l}\text { Rosca Direta } \\
\text { no Crossover }\end{array}$ & $1.87 \pm 6.47$ & $\begin{array}{c}25.27 \pm \\
4.98\end{array}$ & $0.001 *$ \\
\hline Mesa Flexora & $\begin{array}{c}22.73 \pm \\
8.82\end{array}$ & $\begin{array}{c}10.10 \pm \\
4.19\end{array}$ & $0.001 *$ \\
\hline
\end{tabular}

Pode-se observar na Tabela 1 que houve diferença significativa na força das idosas que praticavam $\mathrm{TF}$ em relação às mulheres que frequentavam a ATI nos aparelhos Tríceps Pulley ( $\mathrm{p}=0,001)$, Rosca Direta no Crossover $(\mathrm{p}=0,001)$, Cadeira Extensora $(\mathrm{p}=0,001)$ e Mesa Flexora $(\mathrm{p}=0,001)$, evidenciando que as idosas do grupo TF apresentaram maior força máxima em todos os exercícios.

\section{DISCUSSÃO}

Programas de exercícios que visem o trabalho de manutenção e aumento de força muscular é de extrema importância para idosas se manterem ativas, tendo relação às condições que podem auxiliar nas atividades de vida diária (AVDs) e atividades instrumentais de vida diária Programas de exercícios que visem o trabalho de manutenção e aumento de força muscular é de extrema importância para idosas se manterem ativas, tendo relação às condições que podem auxiliar nas atividades básicas de vida diária (ABVDs) e atividades instrumentais de vida diária (AIVDs), assim como, se beneficiar da própria saúde trazendo qualidade de vida para esta população (AGUIAR et al. 2014). Segundo Raso et al. (1997) existe a necessidade das pessoas idosas em praticar exercício físico resistido, para minimizar e prevenir a redução da força muscular. No estudo de revisão bibliográfica de Panisseti et al. (2012) de artigos científicos, publicados entre os anos 2000 a 2011 (janeiro) na plataforma de periódicos do Scielo e Medline, todos os artigos mostraram a progressão da força muscular em idosos quando submetidos ao treinamento resistido com peso livre ou com aparelhos. No presente estudo, quando comparados os resultados da força máxima em idosas no Tríceps Pulley, foram encontradas diferenças significativas entre os dois grupos, dando melhor significância nos resultados para as idosas praticantes de exercícios resistido, provavelmente pela 
possibilidade de se incrementar cargas extras, aplicando o princípio da sobrecarga no treino. O estudo do treinamento de força máxima envolveu membros inferiores e superiores, focando nos grupos musculares mais usados pelos idosos para executar suas AVDs. Com os resultados encontrados no presente estudo, reforçam a afirmativa de que, mulheres idosas podem e devem ser inseridas em programas de treinamento de força, de preferência envolvendo grandes grupos musculares que objetivem o desenvolvimento de força muscular, massa óssea, equilíbrio, flexibilidade e coordenação afim de melhorar as habilidades nas AVDs e diminuição da sarcopenia (AGUIAR et al., 2014).

Em estudos desenvolvidos por Morais et al. (2004) idosas praticantes de musculação que procuravam fazer uma atividade de força para melhorar a qualidade de vida, apresentaram no préteste uma média de 32,2 quilos para força máxima no músculo tríceps, parecido com a força de idosas ativas deste estudo, que frequentam a ATI e apresentaram média de

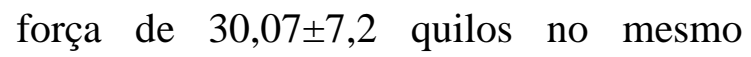
exercício. Após o teste, as idosas do estudo de Morais et al. (2004) passaram a fazer um treinamento de força de 16 semanas e ao final dessa intervenção um segundo teste de carga máxima foi aplicado, obtendo uma média de 38,7 $\pm 4,4$ quilos, que muito se aproxima dos valores encontrados por este estudo, onde mulheres praticantes da musculação apresentaram força média nesse exercício de $40,73 \pm 9,7$.

Estudos realizados por Raso et al. (1997) apresentam dados do pré teste onde a média da força no exercício rosca direta foi de 19,5 \pm 3,9 quilos para idosas saudáveis e fisicamente ativas que foram convidadas a praticar exercícios físicos durante 12 semanas e a média apresentada por essas indivíduas, no mesmo teste de força para bíceps aumentou para 24,5 \pm 3,3 quilos, o que representa um aumento de $25,6 \%$ após o treinamento de força. Neste estudo, o percentual de diferença entre idosas que frequentam a ATI e idosas praticantes de musculação tradicional, no teste de Rosca direta, foi de $26,11 \%$, muito próximo da evolução apresentada pelos estudos de Raso et al. (1997), quando as idosas foram submetidas ao treino de força, sugerindo o aumento da força muscular, nesse exercício, caso as idosas frequentadoras da ATI se submetessem ao mesmo treinamento resistido, haja vista que a força na rosca direta de idosas da ATI é de 25,27 \pm 4,9 quilos e das praticantes de musculação tradicional foi de 31,87 6,4 quilos.

Estudos realizados por Silva et al. (2006) apresentaram dados no treinamento de extensão de joelho com média máxima 
de 25,3 $\pm 8,4$ quilos, para indivíduas que procuravam qualidade de vida sem nenhum tipo de treinamento especifico, após o teste foram submetidas a um treinamento de 12 semanas, apresentando um aumento de $33,7 \pm 9,2$ quilos no máximo de carga levantada, o que representa um ganho de $36,5 \%$ de força máxima após o treinamento. Neste estudo, o percentual de diferença entre idosas que frequentam a ATI e idosas praticantes de musculação tradicional, no teste de extensão de joelho, foi de $43,01 \%$, mais alto que a evolução de força apresentada nos estudos de Silva et al. (2006) cujo tempo de intervenção foi de apenas 12 semanas, sendo que neste estudo as idosas praticam o treinamento de força por mais de 24 semanas, o que parece justificar um percentual de evolução mais elevado.

Silva et al. (2006) também realizaram estudos sobre a força nos flexores de joelho, obtendo média pré teste de $12,7 \pm 4,3$ quilos para força máxima em individuas não treinadas que só procuram qualidade de vida, chegando perto das idosas ativas deste estudo, praticantes de ATI com média de força de 10,10 $\pm 4,19$ quilos. Após o teste, as mesmas indivíduas foram submetidas a um treinamento de força de 12 semanas, e então um segundo teste de força nos flexores foi aplicado, obtendo uma média de $16,7 \pm 5,3$ quilos, muito abaixo do que apresentou este estudo, onde idosas que praticavam a musculação tradicional obtiveram media de 22,73 $\pm 8,82$ quilos. A diferença na evolução da força nos dois estudos se deve, provavelmente ao tempo de prática no treinamento, tendo em vista que as idosas deste estudo praticavam o treinamento de força há mais tempo que as idosas estudadas pelos autores supracitados.

O estudo apresenta limitações no que se refere ao não conhecimento do nível de atividade física real das idosas do Grupo ATI, fato que pode contribuir para o resultado de menor força muscular máxima do que das idosas do Grupo TF, além da ausência de um grupo de idosas sedentárias ou irregularmente ativas para comparação de resultados. Porém, mesmo com as limitações apontadas, espera-se que o presente estudo possa contribuir para maior conhecimento dos profissionais de saúde a respeito da importância da indicação para prática de exercícios resistidos para saúde do idoso.

\section{CONCLUSÃO}

Conclui-se que é significativamente maior a força muscular máxima de idosas praticantes de exercícios resistidos do que de idosas usuárias das ATIs, não praticantes de exercício físico. Sugere-se o estímulo e aderência de idosas em um programa treinamento orientado, estruturado, organizado e planejado para a 
manutenção da independência e habilidades físicas durante o processo de envelhecimento.

\section{REFERÊNCIAS}

AGUIAR, P. P. L. et al. Avaliação da influência do treinamento resistido de força em idosos. Kairós Gerontol, v. 17, n. 3, p. 201-217, 2014. Disponível em: file:///F:/Downloads/22153-56893-1SM.pdf Acesso em: 12 de Novembro de 2016.

BRITO, F. C. E.; LITVOC, C. J. Prevenção e promoção de saúde. São Paulo: Atheneu; 2004. p.1-15.

CAETANO, L. M. O Idoso e a Atividade Física. Rev Educac Fís Desp, v. 11, n. 124, p. 20-28, 2006. Disponível em: http://www.edifesp.fespmg.edu.br/index.ph $\mathrm{p} / \mathrm{scientae} /$ article/view/116/98 Acesso em: 12 de Novembro de 2016.

FREITAS, E. V.; KOPILLER, D. Tratado de Geriatria e Gerontologia. 2a ed. Rio de Janeiro: Guanabara Koogan; 2006. cap. 58, p. 586-59.

GUEDES, D. P.; GUEDES, J. E. P. Manual prático para avaliação em educação física. Barueri: Manole, 2006.

MARTIN, F. G.; NEBULONI, C. C.; NAJAS, M. S. Correlação entre estado nutricional e força de preensão palmar em idosos. Rev Bras Geriatr Gerontol, v. 15, n. 3, p. 493-504, 2012. Disponível em: http://www.scielo.br/scielo.php?script=sci arttext\&pid=S1809-98232012000300010

Acesso em: 12 de Novembro de 2016.

MATSUDO, S.M. et al. Impacto do envelhecimento nas variáveis antropométricas, neuromotoras e metabólicas da aptidão física. Rev Bras

Ciên Mov, v. 8, n. 4, p. 21-32, 2000.
Disponível em:

https://portalrevistas.ucb.br/index.php/RB CM/article/viewFile/372/424 Acesso em: 12 de Novembro de 2016.

MORAIS, I. J. et al. Melhora da força muscular em idosas através de um programa de treinamento de força de intensidade progressiva. Rev Educ Física/UEM, v. 15, n. 2, p. 7-15, 2004. Disponível em: file:///F:/Downloads/34169446-1-PB.pdf Acesso em: 12 de Novembro de 2016.

PADOIN, P. G. et al. Análise comparativa entre idosos praticantes de exercícios físicos e sedentários quanto ao risco de quedas. O Mund Saúde, v. 34, n. 2, p. 156-164, 2010. Disponível em: http://www.saocamilosp.br/pdf/mundo_saude/75/158a164.pdf Acesso em: 12 de Novembro de 2016.

PANISSET, J. Á. et al. Exercício físico resistido: um fator modificável na sarcopenia em idosos. Estud interdiscipl envelhec, v. 17, n. 2, p. 293-304, 2012. Disponível em: file:///F:/Downloads/20706-157407-2PB.pdf Acesso em: 12 de Novembro de 2016.

PRADO, R. A.; TEIXEIRA, A. L. C.; LANGA, C. J. S. O. A influência dos exercícios resistidos no equilíbrio, mobilidade funcional e na qualidade de vida de idosas. O Mund Saúde, v. 34, n. 2, p. 183-191, 2010. Disponível em: http://www.saocamilosp.br/pdf/mundo_saude/75/183a191.pdf Acesso em: 12 de Novembro de 2016.

RASO, V. et al. Exercícios com peso para mulheres idosas: Rev Bras Ativ Fisic

Saúde, v. 2, n. 4, p. 17-24, 1997.

Disponível em: file:///F:/Downloads/11381844-1-PB.pdf Acesso em: 12 de Novembro de 2016.

SILVA, C. M. et al. Efeito do treinamento 
com pesos, prescrito por zona de repetições máximas, na força muscular e composição corporal em idosas. Rev Bras

Cineantropom Desempenho Hum, v. 8, n. 4, p. 40-45, 2006. Disponível em:

https://periodicos.ufsc.br/index.php/rbcdh/ article/viewFile/3924/16754 Acesso em: 12 de Novembro de 2016.

WIECHMANN, M. T.; RUZENE, J. R. S.;
NAVEGA, M. T. O exercício resistido na mobilidade, flexibilidade, força muscular e equilíbrio de idosos. ConScientiae Saúde, v. 12, n. 2, p. 219-226, 2013. Disponível em:

http://www.redalyc.org/pdf/929/92928018 006.pdf Acesso em: 12 de Novembro de 2016. 